\title{
Conductas y actitudes que determinan la duración del desempleo: influencia en desempleados mayores de 45 años de la Región de Murcia, España
}

\author{
Rafael Piqueras Gómez ${ }^{1}$, Tomás Izquierdo Rus² \& Alberto Rodríguez Morejón ${ }^{3}$
}

Recibido:29/08/2018 Aceptado: 10/04/2019

DOI: $10.21772 /$ ripo.v37n2a01

\begin{abstract}
Resumen
Diferentes estudios destacan las dificultades de los adultos para obtener trabajo. En este se propuso conocer la influencia de variables cognitivas y conductuales observando el tiempo que tardan en conseguir trabajo 5771 personas mayores de 45 años en la Región de Murcia, España. Se utilizó el análisis de supervivencia para modelizar el tiempo hasta el empleo según tres escalas: una medida de las expectativas implicadas en el Optimismo Fundado de Búsqueda de Empleo (OFBE), una medida general del Sistema Facilitador de Inserción (SFI) y un Inventario de Conductas de Búsqueda de Empleo (ICBE). Se encontró que las personas optimistas (OFBE alto) pasan 12 meses menos en desempleo. Así mismo, puntuaciones altas en el SFI acortaron el desempleo 19 meses. La búsqueda activa de empleo (ICBE alto) mostró reducir el desempleo en 13 meses. También se observó mayor riesgo de desempleo prolongado para las mujeres y las personas con estudios superiores. La fortaleza de factores cognitivos y conductuales implicados en la búsqueda de empleo puede disminuir la permanencia en desempleo de los mayores de 45 años más de año y medio.
\end{abstract}

Palabras clave: Psicología ocupacional; orientación profesional; motivación; expectativas; optimismo; análisis de supervivencia.

\section{Behaviors and attitudes that determine the duration of unemployment: influence on unemployed over 45 years of age in the Region of Murcia, Spain.}

\begin{abstract}
Different studies highlight the difficulties of adults to obtain work. The aim of this research was to know the influence of cognitive and behavioral variables observing the time it took to get a job to 5771 older than 45 years in the Region of Murcia -Spain. Survival analysis was used to model the time to employment according to three scales: a measure of the expectations implied in the Founded Optimism of Job Search (OFBE), a general measure of the Insertion Facilitator System (SFI) and an Inventory of Job Search Behaviors (ICBE). Optimistic people (high OFBE) spent 12 months less in unemployment. High scores in the SFI cut unemployment by 19 months. The active job search (ICBE high) reduced unemployment in 13 months. There was also a higher risk of prolonged unemployment for women and people with higher education. The strength of cognitive and behavioral factors involved in the search for employment can reduce the permanence in unemployment of those over 45 more than a year and a half.
\end{abstract}

Keywords: Occupational psychology; professional orientation; motivation; expectations; optimism; survival analysis.

\footnotetext{
1 Ms. Interuniversitario en Orientación e Intermediación Laboral. Técnico Superior , Junta de Castilla y León. E-mail: rafaelpiqueras@gmail.com

2 Dr. en Pedagogía y Psicología (2005) y Doctor Internacional en Psicología y Ciencias de la Salud (2014). Profesor Titular. Universidad de Murcia. Facultad de Educación. Departamento de Métodos de Investigación y Diagnóstico en Educación. E-mail: tomasizq@um.es

3 Dr. en Psicología. Profesor Titular. Universidad de Málaga. Facultad de Psicología. Departamento de Personalidad, Evaluación y Tratamiento Psicológico. E-mail: arm@uma.es

Como citar: Piqueras Gómez, R.; Izquierdo Rus, T. \& Rodríguez Morejón, A. (2019). Conductas y actitudes que determinan la duración del desempleo: influencia en desempleados mayores de 45 años de la Región de Murcia, España. Revista Interamericana de Psicología Ocupacional, 37(2), 77-92. DOI: 10.21772/ripo.v37n2a01
} 


\section{Introducción}

La crisis financiera iniciada en 2007 produjo la peor situación de desempleo a nivel mundial desde la Gran Depresión del 29. En España, durante la crisis, la tasa de desempleo pasó del $6.6 \%$ en 2007 al $21.6 \%$ en 2013 (Consejo Económico y Social, 2014). En el cuarto trimestre de 2018, había 3,304,300 personas buscando empleo en España, de las cuales el 38\% tenía más de 45 años y, de éstas, el $62 \%$ llevaba más de un año sin trabajar (Instituto Nacional de Estadística, 2018).

Un paso necesario para afrontar esta situación es conocer las variables más influyentes: qué hace que las personas permanezcan sin trabajo $\mathrm{y}$, por lo tanto, qué elementos habría que cambiar para que la salida hacia el empleo se tornara más probable. Para ello es preciso contar con modelos teóricos potentes basados en la evidencia. En el ámbito de la búsqueda de empleo se han desarrollado distintos modelos explicativos, principalmente desde los enfoques conductual y cognitivo.

Diversos estudios subrayan la influencia de los elementos conductuales implicados en la búsqueda de empleo en los resultados de inserción y la duración del desempleo (Viáfara \& Uribe, 2009). Entre ellos, destacan las intervenciones grupales como los clubs de empleo (Mallinckrodt \& Fretz, 1988; Potocnik \& Sonnentag, 2013; Strauser \& Berven, 2006) o el entrenamiento en habilidades para aumentar la autoeficacia (Fort, Jacquet, \& Leroy, 2011; Vuori \& Vinokur, 2005)

Los enfoques cognitivos estudian la actitud - entendida como la tendencia a la acción o evitación frente a un objetivo determinado - como un elemento central para explicar la conducta. En el proceso de búsqueda, algunos autores destacan el impacto de los factores motivacionales en la permanencia en desempleo (Latham, 1987; Tøge \& Blekesaune, 2015), lo que se asocia frecuentemente con un aumento de la ansiedad (Alconada, 2003) o con la procastinación de la conducta de búsqueda (Lay \& Brokenshire, 1997). Se observa la influencia de elementos como las expectativas (Piqueras, Rodríguez, \& Rueda, 2008), la asertividad (Schmit, Amel, \& Ryan, 2006), los objetivos de búsqueda (Creed, King, Hood, \& McKenzie, 2009), la resiliencia (López, 2007) o la actitud proactiva (Cai et al., 2015). A menudo, estos enfoques explican la búsqueda de empleo desde la teoría de la conducta planeada (Ajzen, 1985; Izquierdo, 2012). En esta línea, destacan los programas dirigidos a mejorar los aspectos personales (Pablo, 1996) que intentan cambiar las actitudes de búsqueda desde una perspectiva muy cercana al concepto de optimismo fundado (Palenzuela, 1989) que hace hincapié en la importancia de las expectativas de autoeficacia, éxito y locus de control, (Caliendo, Cobb-Clark, \& Uhlendorff, 2015; Fort et al., 2011; Hernández, Ramos, Negrín, Ruiz, \& Hernández, 2011 Plumly \& Oliver, 1987).

A pesar de que algunos estudios de resultados destacan un mejor comportamiento de los enfoques cognitivos frente a los conductuales (Aramburu, 2003), las investigaciones demuestran que es mejor combinar técnicas de orientación conductual y cognitiva que aplicar una sola de las dos (Liu, Huang, \& Wang, 2014). Esta línea de integración ha dado lugar al desarrollo de buenas prácticas por parte de los técnicos de los Servicios Públicos de Empleo en España (Almagro, 2017), y a una mejor conceptualización de su trabajo a partir de la aplicación del denominado Sistema Facilitador de Inserción (SFI) que se muestra en la figura 1. Se trata de un esquema de los cuatro elementos fundamentales implicados en el proceso de búsqueda de empleo: la planificación, la información, las habilidades y la motivación (Montilla, 2003;2000a Piqueras, 2010). 
La figura 1 representa una lectura sistémica (Bertalanffy, 1993; Piqueras \& Rodríguez, 1997) que se ajusta a los presupuestos del Construccionismo Social (McNamee \& Gergen, 1996; Pakman, 1997), incorporando los significados y cogniciones socialmente construidos como elementos clave del proceso de búsqueda de empleo. Su estrategia fundamental de intervención es la denominada Centrada en las Soluciones (Altuna, 2005; O'Hanlon \& WeinerDavis, 1990; Piqueras \& Rodríguez, 1997; Piqueras, 2003), muy extendida en la psicología actual (Rodríguez, 2017) y que ha servido de referencia para los principales manuales y guías de trabajo con personas desempleadas (Alconada, Carbonero, González, Lucas, \& Piqueras, 2003; Piqueras \& Rodríguez, 1997; Servicio Regional de Empleo y Formación, 2007).

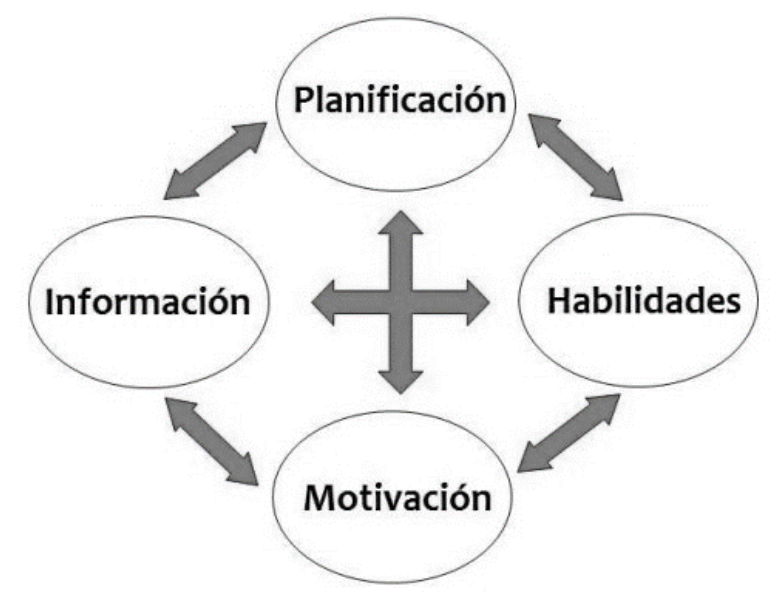

Figura 1. Representación gráfica del Sistema Facilitador de Inserción.

Por otra parte, diversos estudios destacan que las consecuencias derivadas del desempleo se agravan con la edad (Álvaro-Estramiana, 1992; Buendía, 2010; Chan \& Huff Stevens, 2001; Hedge \& Borman, 2012; Gallo, Bradley, Siegel, \& Kasl, 2000; Gutierrez, 2016; Izquierdo \& Alonso, 2010; Izquierdo \& López, 2013; Izquierdo, 2015). Existe una relación directa entre la edad y la duración del desempleo (Piqueras, Rodríguez \& Rueda, 2008), algo que a partir de los 45 años hace disminuir las relaciones sociales favoreciendo la exclusión
(Escarbajal, Izquierdo, \& López, 2014; Izquierdo, 2012). Un problema añadido es que las personas de más edad están menos dispuestas a participar en las actividades de formación (Amber Montes, 2016; Ng \& Feldman, 2012) y disminuyen sus conductas de búsqueda de empleo (Zacher et al., 2013).

Se hacen necesarios estudios específicos que aborden el problema del desempleo de las personas mayores de 45 años (Hedge \& Borman, 2012) y proporcionen instrumentos de evaluación y toma de decisiones adecuados para intervenir con eficacia en situaciones de pérdida de trabajo o reentrada al mercado laboral.

El objetivo del presente trabajo fue conocer el grado de influencia de distintas variables medidas de forma objetiva en el resultado de búsqueda de empleo para las personas mayores de 45 años que perdieron el trabajo durante la crisis, con el fin de facilitar el diseño de intervenciones más eficaces.

\section{Metodología}

Se usó una metodología adaptada a los recursos y medios técnicos disponibles en los servicios de empleo, más concretamente en el Servicio Público de Empleo y Formación de la Región de Murcia (SEF). Se destaca la utilización de la base de datos registrados y el uso de análisis gráficos predictivos de la permanencia en desempleo.

\section{Participantes}

Se analizan los datos de una muestra incidental de 5,771 personas, mayores de 45 años inscritas en el SEF que habían iniciado una intervención de orientación para el empleo entre los años 2010 y 2014 en la que fueron invitados a cumplimentar los tres instrumentos de medida al inicio del programa.

Se conocen datos relativos a su edad, sexo y nivel formativo. Se contabilizan 2,956 hombres (51\%) y 2815 mujeres (49\%), con una edad 
media de 51.01 años, desviación típica DT $=4.03$ y rango $=45-71$. Tan sólo 334 del total $(5.8 \%)$ tenían estudios universitarios.

\section{Instrumentos}

Se valoran los tres cuestionarios integrados en el sistema informático del SEF que se describen a continuación:

\section{Optimismo Fundado de Búsqueda} de Empleo (OFBE): La escala es una parte del cuestionario de Expectativas de Control Percibido de Búsqueda de empleo de 12 ítems que cuenta con datos de fiabilidad específicos con un coeficiente alfa de Cronbach de .80 (Piqueras, Rodríguez \& Palenzuela, 2016). Incluye nueve ítems que se evalúan con una escala tipo Likert de nueve puntos que solicitan el nivel de acuerdo con una frase desde el 1 "Nada de acuerdo" hasta el 9 "Muy de acuerdo". Los ítems se dividen a su vez en tres subescalas con tres ítems cada una: autoeficacia de búsqueda de empleo, éxito de búsqueda de empleo y locus de control interno de búsqueda de empleo. Estas tres subescalas son las referidas a las expectativas de carácter positivo que se corresponden con la definición de "optimismo fundado" de Palenzuela $(1989,1988)$.

Sistema Facilitador de Inserción (SFI): Es un cuestionario de seis ítems, de respuesta en escala tipo Likert de nueve puntos que solicitan el nivel de acuerdo con una afirmación desde el 1 "No, completamente en desacuerdo" hasta el 9 "Si, completamente de acuerdo". Está diseñado para obtener información sobre los cuatro elementos del sistema facilitador de inserción:

- Planificación: un ítem sobre la capacidad del usuario para organizar su búsqueda de empleo.

- Información: un ítem para establecer si la persona tiene información adecuada para guiar su búsqueda de empleo.

- Habilidades: un ítem que evalúa la creencia que la persona tiene sobre su competencia en el desempeño de las técnicas de búsqueda de empleo.

- Motivación: contiene tres ítems que evalúan el nivel de ánimo para buscar trabajo, las expectativas de éxito y la posición del trabajo en la escala de valores.

Inventario de conductas de búsqueda de empleo (ICBE): Es un registro de los diferentes tipos de acciones dirigidas a la búsqueda de empleo. El inventario se estructura en un primer grupo de ocho ítems que recoge los tipos de conductas de búsqueda más habituales y su frecuencia en cuatro niveles: ocasional, mensual, semanal y diaria; un segundo bloque de tres elementos valora la forma de organizar y planificar de la búsqueda de empleo y un ítem final mide la intensidad de búsqueda codificado según el número de horas dedicados a la búsqueda de empleo, en cuatro bloques temporales, de 1 a 5 , de 5 a 1 , de 10 a 20 o más de 20 horas a la semana.

Estos dos cuestionarios (SFI e ICBE) fueron construidos por técnicos expertos del SEF y utilizados ampliamente en su trabajo, aunque no disponen de estudios de fiabilidad y validez publicados.

\section{Procedimiento}

En el año 2015 se propuso al SEF un estudio de los resultados obtenidos por los usuarios de orientación mayores de 45 años atendidos desde enero de 2010 a diciembre de 2014. Esto para evaluar la eficacia del servicio y la utilidad de los datos recogidos en sus bases de datos. Desde servicio de informática del SEF se extrajeron los datos de los tres cuestionarios utilizados como instrumentos de medida, así como el resto de los datos sociodemográficos de los participantes (sexo, edad, nivel académico). Posteriormente, se cruzó esta información con la base de datos del registro de contratos del Servicio Público de Empleo Estatal con el objeto de conocer si habían conseguido un puesto de trabajo durante el periodo de estudio. 


\section{Análisis estadístico}

Se aplicó el procedimiento de análisis de supervivencia (Cleves, Gould, Gutierrez, \& Marchenko, 2008; Jenkins \& García-Serrano, 2004) que pretende modelizar el riesgo hasta que se produzca un determinado suceso y el efecto que distintas covariables tienen en su consecución. Al contrario de otros métodos de análisis, se pueden utilizar datos incompletos o censuras (Klein \& Goel, 1992; Klein \& Moeschberger, 2003). Así, aquellos casos en los que no se conozca el tiempo total hasta la ocurrencia del suceso (la consecución de un contrato en este caso) son también tenidos en cuenta en el análisis.

Se utilizó el procedimiento de regresión de Cox (Harrell, 2015; Santabárbara \& Rubio, 2016) que permite modelar el riesgo de la ocurrencia de un suceso en cada instante del tiempo en función de distintas covariables. Se usó el paquete estadístico SPSS en su opción de análisis de supervivencia / regresión de Cox. Las variables utilizadas en el análisis fueron:

Variable tiempo: Permanencia. Días transcurridos desde que se inició la orientación hasta la consecución de un contrato o hasta la fecha final de observación (censuras). Se tomó como referencia inicial la fecha de aplicación de las escalas que se realiza al inicio de la orientación y como final la de contratación o la extracción informática de los datos que se realizó el 4 de mayo de 2015.

Variable estado: Contrato inicial. Identifica si la ocurrencia del suceso ha tenido lugar. Toma el valor "S" si ha obtenido un contrato tras la aplicación de la escala y " $\mathrm{N}$ " en caso contrario.

Covariables. Son las variables que se consideraron relevantes o influyentes en la permanencia en desempleo. Se analizaron las tres escalas que se corresponden con las puntuaciones obtenidas en las escalas SFI, ICBE y OFBE. Por otro lado se exploraron otras variables que tienen una amplia presencia en los estudios relacionados con el desempleo: el sexo y posesión estudios universitarios.

Un requisito para poder aplicar este análisis es que las variables cumplan el supuesto de impactos o riesgos proporcionales (Beyersmann, Wolkewitz, \& Schumacher, 2008). Esto quiere decir que el cociente de impactos debe ser aproximadamente constante a lo largo del tiempo. Esto se puede comprobar de manera gráfica observando en la función de impacto cómo las líneas de los distintos valores de las variables se disponen de forma paralela manteniendo una distancia aproximadamente constante en la línea temporal.

Al tratarse de un modelo de regresión, se analizó la significación de las variables que a introducir en la ecuación para verificar su capacidad predictiva. Se construyó un modelo por pasos con las variables que entran en la ecuación.

Finalmente se calculó el riesgo de permanencia prolongada en desempleo, entendido como la probabilidad de permanecer más de un año sin conseguir un contrato de trabajo. Estos valores se extrajeron mediante la opción análisis de supervivencia / función de línea base que permite obtener el riesgo de permanecer en desempleo para cada momento temporal deseado.

\section{Resultados}

De los 5771 casos disponibles, se excluyeron del análisis 445 casos $(7.7 \%)$ con valores perdidos. En 2496 casos (43.3\%) ocurrió el evento (contrato) y 2830 casos (49.0\%) son datos censurados que permanecían en desempleo en el momento final de extracción de los datos.

Se verificó que las variables estudiadas se ajustaran al supuesto de impactos proporcionales mediante la construcción de las gráficas de la función de impacto mostradas en el apéndice A, donde se observa que el riesgo evoluciona de forma aproximadamente paralela. 
Una vez comprobado que todas las variables entraran en la ecuación, se recodificaron los datos de las escalas en puntuaciones altas o bajas (por encima o por debajo de la media) para construir dos líneas de supervivencia. La tabla 2 muestra las medias y las desviaciones típicas de las tres escalas.

Tabla 1. Variables en la ecuación
En las variables continuas, se representaron los datos correspondientes a cada uno de sus cuartiles y en las nominales, los datos de cada grupo: hombres / mujeres y estudios universitarios SI/NO.

Utilizando el método adelante por pasos (Wald), tal y como puede observarse en la tabla 1 , las 5 variables propuestas fueron admitidas en la ecuación al nivel de confianza $p<.05$.

\begin{tabular}{llllcllc}
\hline & & B & ET & Wald & gl & Sig. & Exp(B) \\
\hline Paso 1 & ICBE & .344 & .030 & 129.188 & 1 & .000 & 1.411 \\
\hline Paso 2 & ICBE & .289 & .031 & 86.986 & 1 & .000 & 1.335 \\
& SEXO & .384 & .041 & 85.598 & 1 & .000 & 1.468 \\
\hline Paso 3 & SFI & .093 & .016 & 33.748 & 1 & .000 & 1.098 \\
& ICBE & .185 & .036 & 26.893 & 1 & .000 & 1.203 \\
& SEXO & .387 & .041 & 87.259 & 1 & .000 & 1.473 \\
\hline Paso 4 & SFI & .094 & .016 & 33.988 & 1 & .000 & 1.098 \\
& ICBE & .190 & .036 & 28.154 & 1 & .000 & 1.209 \\
\hline \multirow{5}{*}{ Paso 5 } & SEXO & .382 & .042 & 84.659 & 1 & .000 & 1.465 \\
& ESTUDSUP & .240 & .091 & 6.917 & 1 & .009 & 1.271 \\
& SFI & .075 & .018 & 16.631 & 1 & .000 & 1.077 \\
& ICBE & .187 & .036 & 27338 & 1 & .000 & 1.206 \\
& OFBE & .039 & .018 & 4.567 & 1 & .033 & 1.040 \\
& SEXO & .380 & .042 & 83.523 & 1 & .000 & 1.462 \\
& ESTUDSUP & .241 & .091 & 6.996 & 1 & .008 & 1.273 \\
\hline
\end{tabular}

Tabla 2. Medias y desviaciones típicas de las escalas.

\begin{tabular}{lcc}
\hline \multicolumn{1}{c}{ Escala } & $M$ & $D E$ \\
\hline SFI & 6.08 & 1.46 \\
ICBE & 2.21 & .65 \\
OFBE & 6.68 & 1.32 \\
\hline
\end{tabular}
probabilidad acumulada $=.5$. 19 meses. supervivencia para cada variable, donde se marca el valor de la permanencia media en el lugar de corte de las líneas de supervivencia para la

La figura 2, muestra que las puntuaciones altas en la valoración general del Sistema Facilitador de inserción, acortaron el desempleo 
Función de supervivencia para modelos 1 - 2

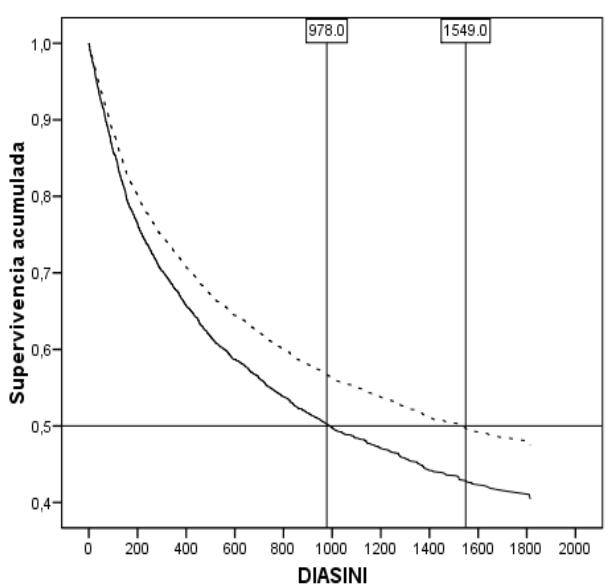

Figura 2. Permanencia en desempleo para puntuaciones SFI en la media.

El riesgo de desempleo prolongado para los adultos que puntuaron alto en SFI fue casi un $6 \%$ menor que para los que puntuaron bajo, tal y como se refleja en la tabla 3.

Tabla 3. Permanencia y riesgo de desempleo prolongado para escala SFI en la media

\begin{tabular}{lcc}
\hline & $\begin{array}{c}\text { Permanencia media } \\
\text { en desempleo (días) }\end{array}$ & $\begin{array}{c}\text { Riesgo de desempleo } \\
\text { prolongado }(\% \text { de } \\
\text { permanencia }>12 \text { meses })\end{array}$ \\
\hline SFI alto & 978 & $68.00 \%$ \\
SFI bajo & 1549 & $73.80 \%$ \\
\hline Diferencia & & \\
para valores & -570 & $-5.80 \%$ \\
altos: & & \\
\hline
\end{tabular}

La figura 3, muestra la probabilidad de permanencia según la actividad de búsqueda de empleo realizada. Puntuar alto en ICBE redujo el tiempo en desempleo 13 meses.

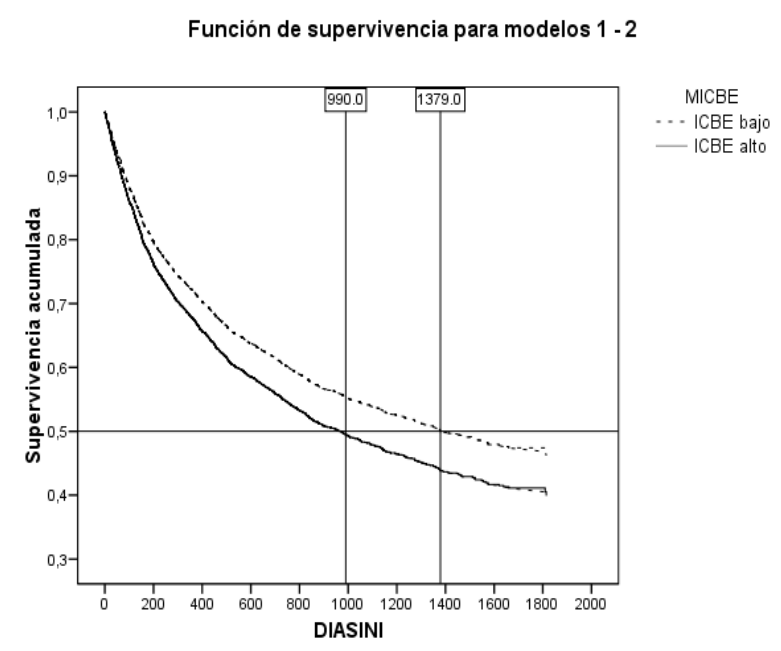

Figura 3. Permanencia en desempleo para puntuaciones ICBE en la media.

En la tabla 4 se evidenció un descenso de cerca de cinco puntos en el riesgo de desempleo prolongado para los adultos activos en la búsqueda.

Tabla 4. Permanencia y riesgo de desempleo prolongado para escala ICBE en la media.

\begin{tabular}{|c|c|c|}
\hline & $\begin{array}{l}\text { Permanencia media } \\
\text { en desempleo (días) }\end{array}$ & $\begin{array}{c}\text { Riesgo de desempleo } \\
\text { prolongado }(\% \text { de } \\
\text { permanencia }>12 \text { meses })\end{array}$ \\
\hline ICBE alto & 990 & $68.20 \%$ \\
\hline ICBE bajo & 1379 & $72.80 \%$ \\
\hline $\begin{array}{l}\text { Diferencia } \\
\text { para valores } \\
\text { altos: }\end{array}$ & -388 & $-4.60 \%$ \\
\hline
\end{tabular}

En la figura 4, se observa que las personas mayores de 45 años con altas expectativas de éxito, autoeficacia y un locus de control interno (OFBE alto), llegaron al empleo mucho antes que los que puntuaron por debajo de la media. 
Función de supervivencia para modelos 1 - 2

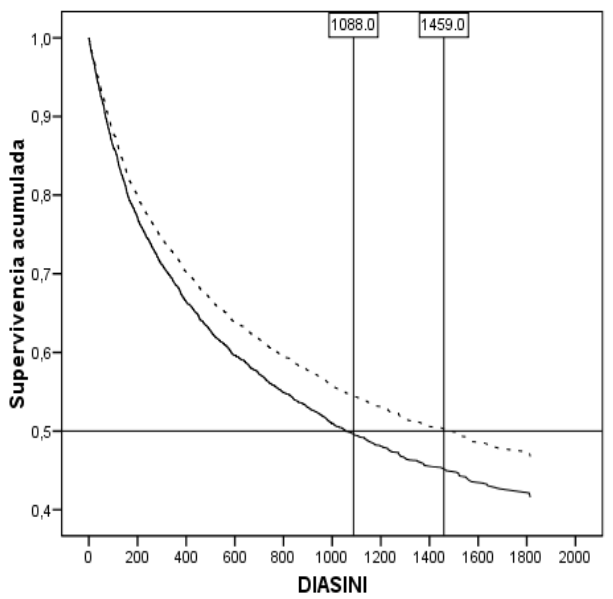

Figura 4. Permanencia en desempleo para puntuaciones OFBE según la media.

En la tabla 5, se observa que las personas adultas optimistas también redujeron un $5 \%$ el riesgo de permanencia prolongada frente a las pesimistas.

Tabla 5. Permanencia y riesgo de desempleo prolongado para escala OFBE en la media.

\begin{tabular}{lcc}
\hline & $\begin{array}{l}\text { Permanencia media } \\
\text { en desempleo (días) }\end{array}$ & $\begin{array}{c}\text { Riesgo de desempleo } \\
\text { prolongado }(\% \text { de } \\
\text { permanencia }>12 \text { meses) }\end{array}$ \\
\hline OFBE alto & 1088 & $68.90 \%$ \\
OFBE bajo & 1459 & $73.70 \%$ \\
\hline Diferencia & & \\
para valores & & $-4.80 \%$ \\
altos: & -371 & \\
\hline
\end{tabular}

A pesar del prolongado seguimiento, no se pudo calcular la permanencia media de las mujeres mayores de 45 años ya que la curva no alcanzó el .05 de probabilidad de inserción como se muestra en el figura 5. Aun así, las mujeres presentaron una permanencia en desempleo claramente superior a los 897 días de los hombres.
Función de supervivencia para modelos 1 - 2

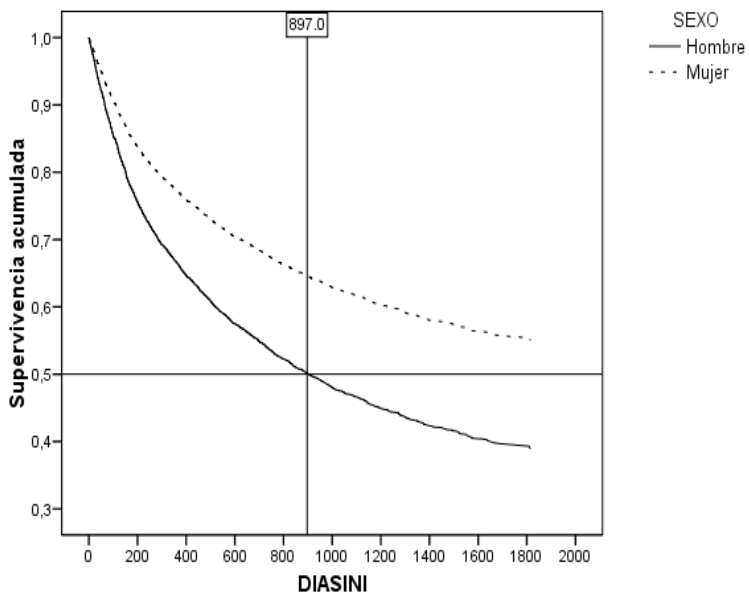

Figura 5. Permanencia en desempleo para mujeres y hombres según la media.

El riesgo de permanencia prolongada de las mujeres fue del $78.8 \%$ frente al $66.35 \%$ de los hombres; más de 12 puntos de diferencia.

Tal y como muestra la figura 6, los mayores de 45 años con estudios universitarios permanecieron más tiempo en desempleo que los que no alcanzaron ese nivel de estudios.

Función de supervivencia para modelos 1 -2

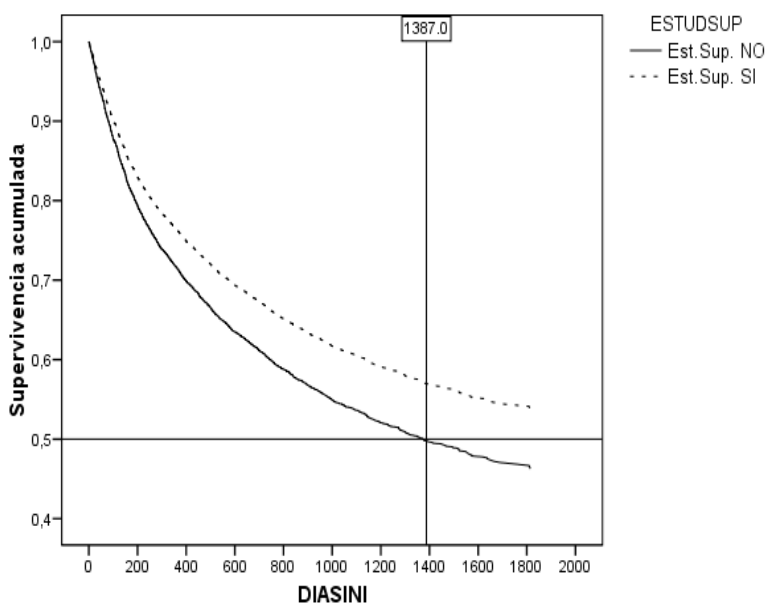

Figura 6. Permanencia en desempleo con o sin estudios superiores según la media. 
También el riesgo de alcanzar el año de desempleo aumentó más de 6 puntos para los desempleados universitarios, pasando del $72.4 \%$ por no tener estudios a un $78.8 \%$ en el caso de tenerlos.

\section{Discusión}

Los resultados mostraron claras diferencias en el tiempo de permanencia en desempleo para todas las variables.

Las personas mayores de 45 años que se informan, manejan bien sus habilidades, se planifican y se enfrentan a la búsqueda de empleo de manera motivada (alto SFI) obtienen empleo, como media, 19 meses antes que las que no lo hacen. Un SFI bajo representa un riesgo de permanencia prolongada en desempleo del 74\%, seis puntos más que el alto. Cobra importancia trabajar la atención a las personas que buscan trabajo de una manera global, incluyendo todos los elementos del Sistema Facilitador de Inserción, tanto los que tienen que ver con la conducta y la información como la motivación y la construcción de objetivos.

Las estrategias de búsqueda de empleo juegan un papel muy importante en la calidad de la inserción (Koen, Klehe, Van Vianen, Zikic, \& Nauta, 2010). El ICBE valora la forma de afrontar la búsqueda, tanto en la realización de distintas actividades de búsqueda como en la intensidad de las mismas. Se pudo observar que quienes presentaron una actividad por debajo de la media permanecieron en desempleo 13 meses más que aquellos que mostraron una búsqueda más activa. Las personas con baja actividad de búsqueda aumentaron el riesgo de convertirse en paradas de larga duración hasta casi el 73\%.

El optimismo que los mayores de 45 años presenten ante la tarea de buscar trabajo juega un papel importante en la consecución de empleo. Tener expectativas altas de éxito y autoeficacia así como considerar que conseguir trabajo dependerá de su esfuerzo (locus de control interno) disminuyó más de un año la búsqueda de empleo. Y, al contrario, ser pesimista aumentó casi un 5\% el riesgo de estar más de 12 meses buscando trabajo. Acciones dirigidas al desarrollo de fortalezas personales (Pablo, 1996) o intervenciones grupales como las denominadas lanzaderas de empleo (Durán, 2016) basadas en la psicología positiva, el coaching o las estrategias de regulación de las emociones (Wang, Xu, Zhang, \& Fang, 2017), representan una opción importante para mejorar las expectativas de los adultos en situación de desempleo prolongado.

A pesar de que la brecha de género ha disminuido los últimos años, la variable sexo sigue mostrado una importancia fundamental en el mercado laboral (Krause, 2013). Esto se observa claramente en mujeres en los tramos de edad más avanzados que en muchas ocasiones, aunque quieran trabajar, se ven abocadas a tareas domésticas relacionados con el cuidado de personas (Neumark, Burn \& Button, 2015). Se observa con claridad que su permanencia en desempleo es mucho más larga que la de los varones: cuando estos alcanzan el $50 \%$ de inserción, el $65 \%$ de las mujeres siguen desempleadas, extendiendo en el tiempo su situación de vulnerabilidad (Llinares, González, Córdoba, \& Zacarés, 2018)

El nivel académico ha sido tradicionalmente asociado a una mejor empleabilidad (Formichela \& London, 2013; Moreno Mínguez, 2015). Esto es muy evidente en los jóvenes; sin embargo, en países como España, para las personas mayores de 45 años, la formación universitaria puede representar un obstáculo para su inserción en un mercado laboral en crisis donde los puestos cualificados suelen recaer en jóvenes mejor formados y menos exigentes en sus remuneraciones.

Actualmente, no son muchos los estudios específicos relativos a personas desempleadas en este tramo de edad (Izquierdo, 2014) ya que una gran parte de las investigaciones han 
centrado su atención en la población general o en los jóvenes en proceso de transición a la vida activa (Aramburu, 1998; Hodkinson, Sparkes, \& Hodkinson, 2013). Al contrario que los jóvenes, los mayores de 45 años son normalmente personas con experiencia que conocen el mercado laboral. Sus actitudes hacia el trabajo son diferentes y pueden mediatizar su incorporación a un nuevo empleo (Izquierdo \& López, 2013). Así mismo, muchas personas con más de 45 años llegan al desempleo con pocas expectativas de recuperar su anterior posición laboral y se sienten desanimadas para buscar trabajo (Gallo et al., 2000; Izquierdo, 2012; Izquierdo \& López, 2013).

\section{Conclusiones}

Los resultados confirman la conveniencia de combinar elementos conductuales y cognitivos (Liu, Huang, \& Wang, 2014) para observar el sistema de búsqueda en su totalidad (Montilla, 2003;2000a Piqueras \& Rodríguez, 1997). Algo que permite el SFI, una medida que se revela como el mejor predictor de inserción de las tres escalas y que puede indicar en qué elementos del sistema podemos apoyarnos para intervenir con mejores probabilidades de éxito.

Impulsar actuaciones que fortalezcan los factores cognitivos y conductuales implicados en la búsqueda de empleo, puede disminuir la permanencia en desempleo de los mayores de 45 años más de año y medio. Las respuestas a las escalas utilizadas y los resultados obtenidos invitan a mejorar e impulsar las actuaciones de orientación profesional, incluyendo el entrenamiento en conductas de búsqueda y la configuración de grupos de activación que mejoren las habilidades sociales, comunicativas y tecnológicas en un ambiente motivado. Esas actuaciones ayudarán a superar las barreras que las empresas presentan a la hora de incorporar a estas personas por su edad, perdiéndose, en muchas ocasiones, importantes capacidades y conocimientos acumulados en largos años de experiencia.

\section{Limitaciones y nuevas líneas de actuación}

Es evidente que este estudio y otros similares cobran un especial interés en contextos laborales como el europeo, donde el envejecimiento de la población activa es cada vez más acuciante. Sería, no obstante, importante extender este conocimiento a otros países con un mercado laboral más joven, para poder actuar de manera preventiva y evitar situaciones de exclusión para los adultos que pierden su empleo.

Es conveniente ampliar los ítems del ICBE y del SFI dirigidos a sondear el uso de las nuevas tecnologías y realizar estudios de fiabilidad y validez que faciliten su aplicación, interpretación y alcance científico.

También las intervenciones que fomentan la proactividad han mostrado evidencias de mejora de la probabilidad de inserción en personas con dificultades relacionadas con la salud y la edad (Brown, Cober, Kane, Levy, \& Shalhoop, 2006; Zacher et al., 2013). Sería una aportación importante añadir nuevos ítems a la escala OFBE que midieran estos aspectos.

\section{Referencias}

Ajzen, I. (1985). From Intentions to Actions: A Theory ofPlanned Behavior.In Action Control (pp. 11-39). Berlin, Heidelberg: Springer Berlin Heidelberg. Doi:10.1007/978-3-64269746-3 2

Alconada, J. (2003). Ansiedad en situaciones de búsqueda de empleo. In Junta de Castilla y León (Ed.), Guía sobre aspectos generales para el desarrollo de las acciones de orientación (pp. 61-100). Valladolid: Junta de Castilla y León. Recuperado de http:// www.empleo.jcyl.es/web/jcyl/Empleo/es/ Plantilla100DetalleFeed/1284216458054/ Publicacion/1185904251175/Redaccion 
Alconada, J., Carbonero, M. Á., González, M. . B., Lucas, S., \& Piqueras, R. (2003). Guía sobre aspectos generales para el desarrollo de las acciones de orientación laboral. Valladolid: Junta de Castilla y León. Recuperado de http://empleo.jcyl.es/web/jcyl/Empleo/es/ Plantilla100DetalleFeed/1284216458054/ Publicacion/1185904251175/Redaccion

Almagro, L. M. (2017). El servicio Andalucía Orienta desde la perspectiva de sus usuarios $y$ personal técnico. Factores facilitadores $e$ inhibidores de buenas prácticas en orientación profesional (tesis doctoral). Universidad de Sevilla. Doi:10.13140/ RG.2.2.29991.80800

Altuna, A. (2005). El rol del orientador: tomas de posición para una orientación laboral más efectiva. Capital Humano: Revista Para La Integración Y Desarrollo de Los Recursos Humanos, 18(192), 109-115. Recuperado de http://pdfs.wke.es/2/6/5/3/pd0000012653. pdf

Álvaro-Estramiana, J. L. (1992). Desempleo y bienestar psicológico. Madrid: Siglo XXI de España Editores.

Amber Montes, D. (2016). Mayores de 45 años en desempleo. Historias silenciadas y desafios de la formación (tesis doctoral). Universidad de Granada. Granada. Recuperado de http:// digibug.ugr.es/handle/10481/43686

Aramburu, L. (1998). Transición a la vida activa: procesos de búsqueda de empleo en una muestra de universitarios madrileños (tesis doctoral). Universidad Complutense de Madrid. Recuperado de https://dialnet. unirioja.es/servlet/tesis?codigo $=14987$

Aramburu, L. (2003). Enfoques cognitivo y conductual: evaluación de dos programas de intervención con desempleados. Psicothema, 15(Número 2), 183-189. Recuperado de http://www.psicothema.com/psicothema. asp?id=1043
Bertalanffy, L. V. (1993). Teoría general de los sistemas: fundamentos, desarrollo, aplicaciones. Ciudad de México: Fondo de Cultura Económica.

Beyersmann, J., Wolkewitz, M., \& Schumacher, M. (2008). The impact of time-dependent bias in proportional hazards modelling. Statistics in Medicine, 27(30), 6439-6454. Doi:10.1002/sim.3437

Brown, D. J., Cober, R. T., Kane, K., Levy, P. E., \& Shalhoop, J. (2006). Proactive personality and the successful job search: A field investigation with college graduates. Journal of Applied Psychology, 91(3), 717726. Doi:10.1037/0021-9010.91.3.717

Buendía, J. (2010). El impacto psicológico del desempleo. Murcia: Universidad de Murcia, Servicio de Publicaciones.

Cai, Z., Guan, Y., Li, H., Shi, W., Guo, K., Liu, Y., ... Hua, H. (2015). Self-esteem and proactive personality as predictors of future work self and career adaptability: An examination of mediating and moderating processes. Journal of Vocational Behavior, 86, 86-94. Doi:10.1016/J.JVB.2014.10.004

Caliendo, M., Cobb-Clark, D. A., \& Uhlendorff, A. (2015). Locus of Control and Job Search Strategies. Review of Economics and Statistics, 97(1), 88-103. Doi:10.1162/ REST_a_00459

Chan, S., \& Huff Stevens, A. (2001). Job Loss and Employment Patterns of Older Workers. Journal of Labor Economics, 19(2), 484521. Doi:10.1086/319568

Cleves, M. A., Gould, W. W., Gutierrez, R. G., \& Marchenko, Y. U. (2008). An introduction to survival analysis using Stata. Stata Press. Recuperado de https://books.google.es/ books?hl=es\&lr=\&id=xttbn0a-QR 8C\&oi=f nd\&pg=PR13\&dq=Survival+Analysis.. + Jen kins\&ots $=$ a_4KDm5D4d\&sig $=\mathrm{iSWCXzFII}$ 
_4a0lhutNEkFUCvNFw\#v=onepage \&q=Su rvival Analysis.. Jenkins\&f=false

Consejo Económico y Social. (2014). La situación sociolaboral de las persona de 45 a 64 años de edad. Retrieved January 22, 2018, from http:// www.ces.es/documents/10180/1558369/ Inf0214.pdf

Creed, P., King, V., Hood, M., \& McKenzie, R. (2009). Goal orientation, self-regulation strategies, and job-seeking intensity in unemployed adults. Journal of Applied Psychology, 94(3), 806-813. Doi:http:// dx.doi.org/10.1037/a0015518

Durán, E. (2016). Las lanzaderas de empleo. Una estrategia de innovación social para la inserción laboral. Retrieved May 9, 2017, from http://uvadoc.uva.es/ handle/10324/21957

Escarbajal, A., Izquierdo, T., \& López, O. (2014). Análisis del bienestar psicológico en grupos en riesgo de exclusión social. Anales de Psicología, 30(2), 541-548. Doi:10.6018/ analesps.30.2.156591

Formichela, M. M., \& London, S. (2013). Empleabilidad, educación y equidad social. Revista de Estudios Sociales, 47, 79-91. Doi:10.7440/res47.2013.06

Fort, I., Jacquet, F., \& Leroy, N. (2011). Selfefficacy, goals, and job search behaviors. Career Development International, 16(5), 469-481. Doi:10.1108/13620431111168886

Gallo, W. T., Bradley, E. H., Siegel, M., \& Kasl, S. V. (2000). Health effects of involuntary job loss among older workers: findings from the health and retirement survey. The Journals of Gerontology. Series B, Psychological Sciences and Social Sciences, 55(3), S13140. Doi:10.1093/GERONB/55.3.S131

Gutierrez, I. A. (2016). Job insecurity, unemployment insurance and on-the-job search. Evidence from older American workers. Labour Economics, 41, 228-245. Doi:10.1016/j.labeco.2016.05.011

Harrell, F. E. (2015). Regression modeling strategies: with applications to linear models, logistic and ordinal regression, and survival analysis. (Springer, Ed.). New York. Recuperado de https://books.google.es/s?hl $=\mathrm{es} \& 1 \mathrm{r}=\& \mathrm{id}=94 \mathrm{RgCgAAQBAJ} \&$ oi $=\mathrm{fnd} \& \mathrm{p}$ $\mathrm{g}=\mathrm{PR} 7 \& \mathrm{dq}=\mathrm{cox}+$ regresion + model $\&$ ots $=\mathrm{ZA}$ m9UnaN5i\&sig=8DCDv47AhhLYF7FHM $\mathrm{HwH} 0 \mathrm{Vb} 6 \mathrm{dt} 4 \# \mathrm{v}=$ onepage $\& \mathrm{q}=\mathrm{cox}$ regresion model $\& \mathrm{f}=$ false

Hedge, J. W., \& Borman, W. C. (2012). The Oxford handbook of work and aging. Oxford University Press. Recuperado de https:// books.google.es/s?hl=es\&lr=\&id=WHdYIE $76 \mathrm{xu} 4 \mathrm{C} \&$ oi $=$ fnd \&pg $=\mathrm{PP} 1 \& \mathrm{dq}=\mathrm{The}+\mathrm{Oxfor}$ $\mathrm{d}+$ Handbook + of + Job + Loss + and + Job + Sear ch\&ots $=\mathrm{Bh} 5388 \mathrm{CHno \& sig}=\mathrm{KDV}$ oewtCv $\mathrm{X} 1 \mathrm{ko} 5 \mathrm{Py} 5 \mathrm{KPxg} 3 \mathrm{bqr} 0 \# \mathrm{v}=$ onepage $\& \mathrm{q}=\mathrm{The}$ Oxford Handbook of Job Loss and Job Search \& $\mathrm{f}=$ false

Hernández, E., Ramos, Y., Negrín, F., Ruiz, C. I., \& Hernández, B. (2011). Empleabilidad Percibida y Autoeficacia para la Búsqueda de Empleo en Universitarios Perception of Employability and Self-Efficacy for Job Seeking in University Students, 27, 2011131. Doi:10.5093/tr2011v27n2a5

Hodkinson, P., Sparkes, A. C., \& Hodkinson, H. (2013). Triumphs and tears: young people, markets and the transition from school to work. Routledge. Recuperado de https://books. google.es/books/about/Triumphs_and Tears.html?id=4PEkAQAAIAAJ\&redir_ $\mathrm{esc}=\mathrm{y}$

Instituto Nacional de Estadística. (2018). Encuesta de Población Activa (EPA): Parados por tiempo de búsqueda de empleo, sexo y grupo de edad. Retrieved March 29, 2019, from https://www.ine.es/jaxiT3/Tabla. htm?t=4111\&L=0 
Izquierdo, T. (2012). Efectos de la duración del desempleo en las actitudes hacia el trabajo de los mayores de 45 años. Revista Electrónica de Investigación $Y$ Docencia (REID), (8), 7-21. Recuperado de https:// revistaselectronicas.ujaen.es/index.php/reid/ article/view/1170

Izquierdo, T. (2014). Análisis de las variables mediadoras que determinan las actitudes hacia el trabajo de los desempleados mayores de 45 años de España y Portugal. Universidad Católica San Antonio de Murcia. Recuperado de https://dialnet.unirioja.es/ servlet $/$ tesis? codigo $=90957$

Izquierdo, T. (2015). Duración del desempleo $\mathrm{y}$ actitudes de los mayores de 45 años en Portugal y España: Un estudio comparativo. Revista de Ciencias Sociales, 21(1), 21-29.

Izquierdo, T., \& Alonso, H. J. (2010). Valores culturales y Consecuencias Psicosociales del Desempleo en América Latina. Revista de Psicología Del Trabajo $Y$ de Las Organizaciones $=$ Journal of Work and Organizational Psychology, 26(2), 123-134. Doi:10.5093/tr2010v26n2a4

Izquierdo, T., \& López, O. (2013). El rol de las actitudes en la inserción laboral de los desempleados mayores de 45 años. Universitas Psychologica, 12(3), 911-922. Doi:10.11144/Javeriana.UPSY12-3.rail

Jenkins, S. P., \& García-Serrano, C. (2004). The Relationship between Unemployment Benefits and Re-employment Probabilities: Evidence from Spain. Oxford Bulletin of Economics and Statistics, 66(2), 239-260. Doi:10.1046/j.0305-9049.2003.00083.x

Klein, J. P., \& Goel, P. K. (1992). Survival Analysis: State of the Art. Springer Netherlands. Recuperado de https://books.google.es/ books? id=xhTvCAAAQBAJ\&dq=klein moeschbergersurvival\&hl=es\&lr\&source $=\mathrm{gbs}$ similarbooks
Klein, J. P., \& Moeschberger, M. L. (2003). Survival analysis: techniques for censored and truncated data. New York: Springer. Recuperado de https://www.amazon.es/dp/ B000YIWEQQ/ref=dp-kindle-redirect? encoding $=\mathrm{UTF} 8 \& \mathrm{btkr}=1$

Koen, J., Klehe, U.-C., Van Vianen, A. E. M., Zikic, J., \& Nauta, A. (2010). Job-search strategies and reemployment quality: The impact of career adaptability. Journal of Vocational Behavior, 77(1), 126-139. Doi:10.1016/J. JVB.2010.02.004

Krause, A. (2013). Don't worry, be happy? Happiness and reemployment. Journal of EconomicBehavior \& Organization, 96, 1-20. Recuperado de http://search.proquest.com/ docview/1504169198? accountid $=14568$

Latham, V. M. (1987). The Job Search Process: An Attitudinal and Behavioral Analysis. Journal of Employment Counseling, 24(1), 7-9. Doi:10.1002/j.2161-1920.1987.tb00216.x

Lay, C. H., \& Brokenshire, R. (1997). Conscientiousness, procrastination, and person-task characteristics in job searching by unemployed adults. Current Psychology, 16(1), 83-96. Doi:10.1007/s12144-997$1017-9$

Liu, S., Huang, J. L., \& Wang, M. (2014). Effectiveness of job search interventions: a meta-analytic review. Psychological Bulletin, 140(4), 1009-41. Doi:10.1037/ a0035923

Llinares, L. I., González, P., Córdoba, A. I., \& Zacarés, J. J. (2018). Women's Job Search Competence: A Question of Motivation, Behavior, or Gender. Frontiers in Psychology, 9, 137. Doi:10.3389/fpsyg.2018.00137

López, S. A. (2007). Efectos Individuales del Despido y la Resiliencia como Facilitador en la Búsqueda de Empleo. Panorama Socioeconómico, 25, 168-173. Recuperado 
de http://panorama.utalca.cl/dentro/2007dic/articulo7.pdf

Mallinckrodt, B., \& Fretz, B. R. (1988). Social support and the impact of job loss on older professionals. Journal of Counseling Psychology, 35(3), 281-286. Recuperado de http://search.proquest.com/ docview/617452114? accountid=14568

McNamee, S., \& Gergen, K. J. (1996). La Terapia como construcción social. Barcelona: Paidós.

Montilla, S. (2003). Orientación profesional para el empleo: un esquema de trabajo multidimensional. Revista de Psicología Del Trabajo Y de Las Organizaciones = Journal of Work and Organizational Psychology, 19(1), 25-57. Recuperado de http://www. redalyc.org/articulo.oa? $\mathrm{id}=231318051002$

Moreno Mínguez, A. (2015). La empleabilidad de los jóvenes en España. Revista Internacional de Investigación En Ciencias Sociales, 11(1), 3-20. Recuperado de https://dialnet.unirioja. es/servlet/articulo?codigo $=5156077$

Neumark, D., Burn, I., \& Button, P. (2015). Is It Harder for Older Workers to Find Jobs? New and Improved Evidence from a Field Experiment. Cambridge, MA. Doi:10.3386/ w21669

Ng, T. W. H., \& Feldman, D. C. (2012). Evaluating Six Common Stereotypes About Older Workers with Meta-Analytical Data. Personnel Psychology, 65(4), 821-858. Doi:10.1111/peps.12003

O’Hanlon, W. H., \& Weiner-Davis, M. (1990). En busca de soluciones: un nuevo enfoque en psicoterapia. Barcelona: Paidos.

Pablo, J. M. de. (1996). Desarrollo de los aspectos personales para la ocupación: una metodología para el cambio con grupos de desempleados. Psychosocial Intervention, 5(15), 75-102.
Pakman, M. (1997). Construcciones de la experiencia humana. Volumen I y II. Barcelona: Gedisa.

Palenzuela, D. L. (1988). Refining the theory and measurement of expectancy of internal vs external control of reinforcement. Personality and Individual Differences, 9(3), 607-629. Doi:10.1016/0191-8869(88)90159-6

Palenzuela, D. L. (1989). Control personal: Un enfoque integrativo-multidimensional. In 2nd Convention of Portuguese Psychologists Association: International Conference. Lisboa, Portugal.

Piqueras, R. (2003). Motivación de búsqueda de empleo. In Junta de Castilla y León (Ed.), Guía sobre aspectos generales para el desarrollo de las acciones de orientación (pp. 101-122). Valladolid: Junta de Castilla y León. Recuperado de http:// www.empleo.jcyl.es/web/jcyl/Empleo/es/ Plantilla100DetalleFeed/1284216458054/ Publicacion/1185904251175/Redaccion

Piqueras, R. (2010). Orientación ocupacional individual. Santo Domingo: Servicio Nacional de Empleo (SENAE). Recuperado de http://www.empleocard.info/centrode-documentacion/guia-de-orientacionocupacional-individual-0

Piqueras, R., \& Rodríguez, A. (1997). Orientación profesional centrada en las soluciones. Psychosocial Intervention, 6(3), 317-338. Recuperado de https://es.slideshare.net/ TxamaMarquesso/orientacin-profesionalcentrada-en-las-soluciones

Piqueras, R., Rodríguez, A., \& Palenzuela, D. L. (2016). Validación de una escala de control personal: una medida específica de las expectativas de control percibido de búsqueda de empleo. Revista de Psicología Del Trabajo Y de Las Organizaciones, 32(3), 153-161. Doi:10.1016/j.rpto.2016.06.001 
Piqueras, R., Rodríguez, A., \& Rueda, C. (2008). Expectativas y duración del desempleo. Revista de Psicología Del Trabajo Y de Las Organizaciones, 24(2), 25-57. Recuperado de http://www.redalyc.org/articulo. oa?id=231316495001

Plumly, L. W., \& Oliver, J. E. (1987). The locus of control attribute and the job search process. Psychological Reports, 61(3), 907-910. Recuperado de http://search.proquest.com/ docview/617512171 ?accountid $=14568$

Potocnik, K., \& Sonnentag, S. (2013). A longitudinal study of well-being in older workers and retirees: : The role of engaging in different types of activities. Journal of Occupational and Organizational Psychology, 86(4), 497-521. Doi:10.1111/ joop. 12003

Rodríguez, A. (2017). Introducción a las psicoterapias: modelos clásicos y contemporáneos. (Amazon Fullfillment, Ed.). Recuperado de https://www.amazon. es/Introducción-las-psicoterapias-clásicoscontemporáneos-ebook/dp/B01N7Y0MJ8

Santabárbara, J., Rubio, E., \& Martinez, T. (2016). Manual de análisis de supervivencia: curvas de supervivencia y regresión de Cox: (ejercicios resueltos con ayuda de IBM SPSS, EPIDAT, OPEN EPI y $R$ ). Zaragoza: Prensas de la Universidad de Zaragoza. Recuperado de http://www.unizar.es/actualidad/ vernoticia_ng.php?id=32500

Schmit, M. J., Amel, E. L., \& Ryan, A. M. (2006). Self-reported assertive job-seeking behaviors of minimally educated job hunters. Personnel Psychology, 46(1), 105-124. Doi:10.1111/j.1744-6570.1993.tb00869.x

Servicio Regional de Empleo y Formación. (2007). Murcia Orienta. Murcia: Servicio
Regional de Empleo y Formación - SEF. Recuperado de http://www.sefcarm.es/web/ a? IDCONTENIDO $=664 \&$ RASTRO $=\mathrm{c} \$ \mathrm{~m} 9$ $983,1251.12595 \&$ IDTIPO $=246$

Strauser, D. R., \& Berven, N. L. (2006). Construction and Field Testing of the Job Seeking Self-Efficacy Scale. Rehabilitation Counseling Bulletin, 49(4), 207-218. Doi:1 0.1177/00343552060490040201

Tøge, A. G., \& Blekesaune, M. (2015). Unemployment transitions and self-rated health in Europe: A longitudinal analysis of EU-SILC from 2008 to 2011. Social Science \& Medicine, 143, 171-178. Doi:10.1016/j. socscimed.2015.08.040

Viáfara, C.A. L., \& Uribe, J. I. G. (2009). Duración del desempleo y canales de búsqueda de empleo en colombia. Revista de Economía Institucional, 2(21), 139-160. Recuperado de https://revistas.uexternado.edu.co/index. php/ecoins/article/view/372/347

Vuori, J., \& Vinokur, A. D. (2005). Job-search preparedness as a mediator of the effects of the Työhön Job Search Intervention on reemployment and mental health. Journal of Organizational Behavior, 26(3), 275-291. Doi:10.1002/job.308

Wang, L., Xu, H., Zhang, X., \& Fang, P. (2017). The relationship between emotion regulation strategies and job search behavior among fourth-year university students. Journal of Adolescence, 59, 139-147. Doi:10.1016/J. ADOLESCENCE.2017.06.004

Zacher, H., Aguinis, H., Beaty, J. C., Boik, R. J., Pierce, C. A., Bal, P. M., ... Winter, G. (2013). Older job seekers' job search intensity: the interplay of proactive personality, age and occupational future time perspective. Ageing and Society, 33(7), 1139-1166. Doi:10.1017/ S0144686X12000451 


\section{Apéndice A}

Gráficas de impactos: comprobación del supuesto de impactos proporcionales.

Se verifica gráficamente que el cociente de impactos tiende a ser constante a lo largo del tiempo (las líneas van separándose de forma paralela). En las escalas: Sistema facilitador de inserción (SFI), Inventario de Conductas de Búsqueda de Empleo (ICBE) y Optimismo Fundado de Búsqueda de Empleo (OFBE), cada línea muestra un cuartil. En la gráfica de SEXO, una línea discontinua para las mujeres y continua para los varones. Para ESTUDIOS SUPERIORES las líneas diferencian: discontinua si tienen estudios superiores o continua si no.
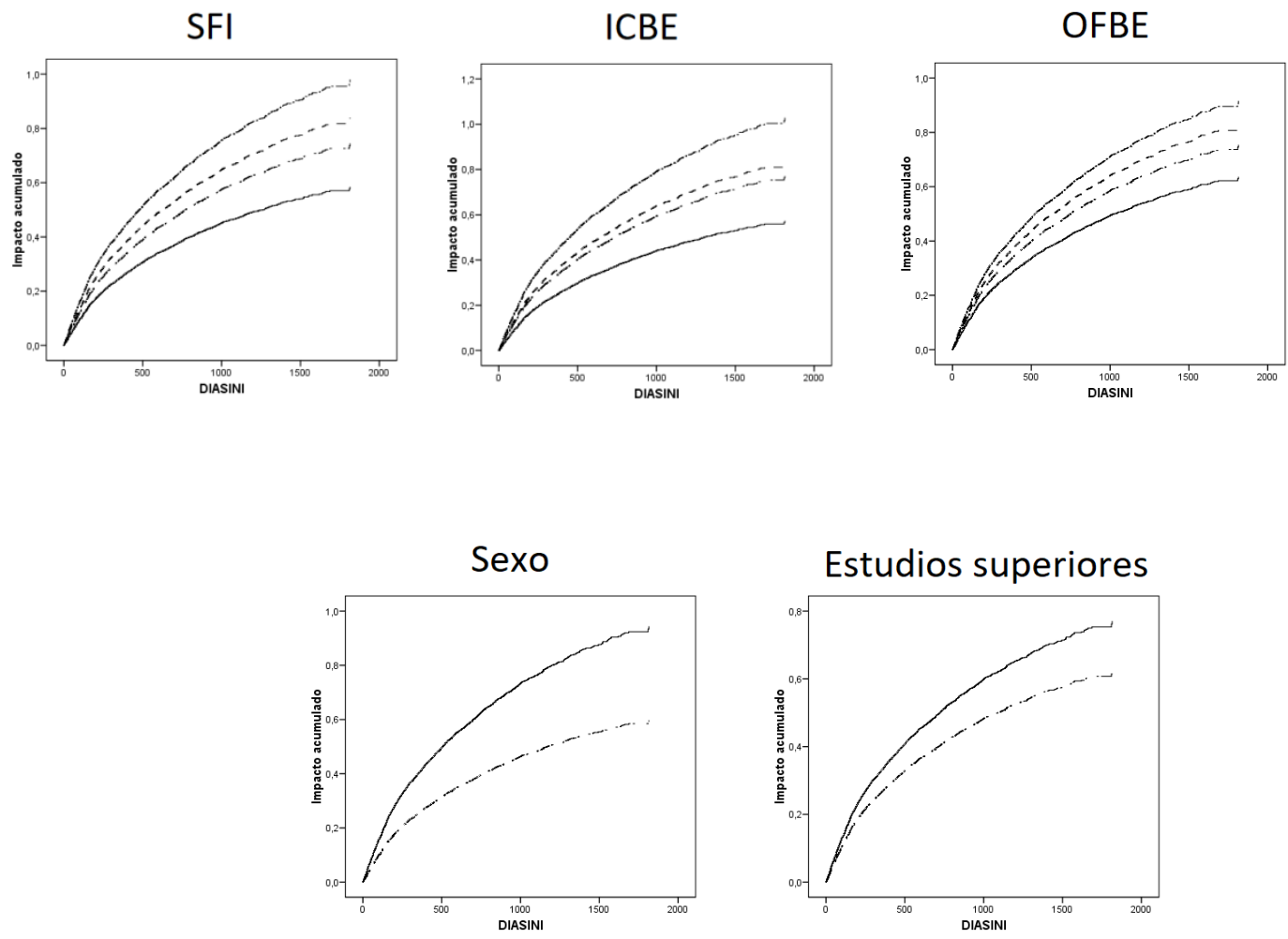